\title{
Robotic Surgery for Giant Presacral Dumbbell-Shape Schwannoma
}

\author{
Farid Yudoyono, ${ }^{1}$ Muhammad Zafrullah Arifin, ${ }^{1}$ Rully Hanafi Dahlan, ${ }^{1}$ Sevline Estethia \\ Ompusunggu, ${ }^{1}$ Shin Dong Ah, ${ }^{2}$ Yi Seong, ${ }^{2}$ Ha Yoon, ${ }^{2}$ Yoon Do Heum, ${ }^{2}$ Kim Keung Nyun ${ }^{2}$ \\ ${ }^{1}$ Neurospine Division, Department of Neurosurgery, Faculty of Medicine, Universitas Padjdajaran-Dr. Hasan \\ Sadikin General Hospital \\ ${ }^{2}$ Departement of Neurosurgery, Yonsei University College of Medicine, Seoul, Korea: Spine and Spinal Cord \\ Research Institute, Yonsei University College of Medicine
}

Abstract
Objective: To demonstrate the feasibility of using da Vinci robotic surgical
system to perform spinal surgery.
Methods: Magnetic resonance imaging (MRI) of a 29-year-old female patient
complaining right pelvic pain for 1 month revealed a 17x8x10 cm non-
homogeneous dumbbell shape encapsulated mass with cystic change located
in the pelvic cavity and caused an anterior displacement of urinary bladder
and colon.
Results: There was no systemic complication and pain decrease 24 hours
after surgery and during 2 years of follow up. The patient started a diet 6
hours after the surgery and was discharged 72 hours after the surgery. The
pathological diagnosis of the tumor was schwannoma.
Conclusions: Giant dumbbell shape presacral schwannomas are rare
tumours and their surgical treatment is challenging because of the complex
anatomy of the presacral. Clinical application of da Vinci robotic surgical
system in the spinal surgical field is currently confined to the treatment of
Received:

\section{Introduction}

Schwannoma, thatisamostlybenign peripheral nerve sheath tumor, arises from the Schwann cells. It grows slowly and asymptomatically in approximately 1 of 40,000 patients admitted in hospitals, most commonly in the age group of 40- and 60-years, with a male to female ratio of $2: 3 .^{1-3}$ The predilection areas include the head and neck; extremities and posterior mediastinum; and retroperitoneoum or pelvis. The presacral space, in particular, is a peculiar anatomic area between the posterior wall of the rectum and the anterior surface of the sacrum, extending upwards to the peritoneal reflection and downwards to the rectosacral

Correspondence:

Farid Yudoyono, Neurospine Division, Department of Neurosurgery, Faculty of Medicine, Universitas

Padjdajaran-Dr. Hasan Sadikin General Hospital

Jl. Pasteur No. 38, Bandung, Indonesia

e-mail: faridspine@gmail.com fascia (Waldeyer). The lateral boundaries are the endopelvic fascia (lateral ligaments), the ureters and the iliac vessels. ${ }^{3-5}$ Schwannoma occuring in this area is known as the presacral schwannoma. There are three types of giant presacral schwannoma based on anatomical findings; the retroperitoneal schwannoma, the intraosseous (intrasacral) schwannoma, and the spinal (dumb-bell) schwannoma. ${ }^{2,6-8}$

During the past decade, robotic surgical systems have continuously been developed. These advances have allowed the substitution of laparoscopic procedures in many surgical fields owing to its 3D vision and enhanced ergonomics. The 6 degrees of freedom, that is provided by the robot instrumentation, in combination with a 3D high-definition image and rock steady operating arms allow neurosurgeons to operate in an extreme view. Furthermore, the magnification offered by the endoscope varies from $12 \mathrm{x}$ to $40 \mathrm{x}$ depending 


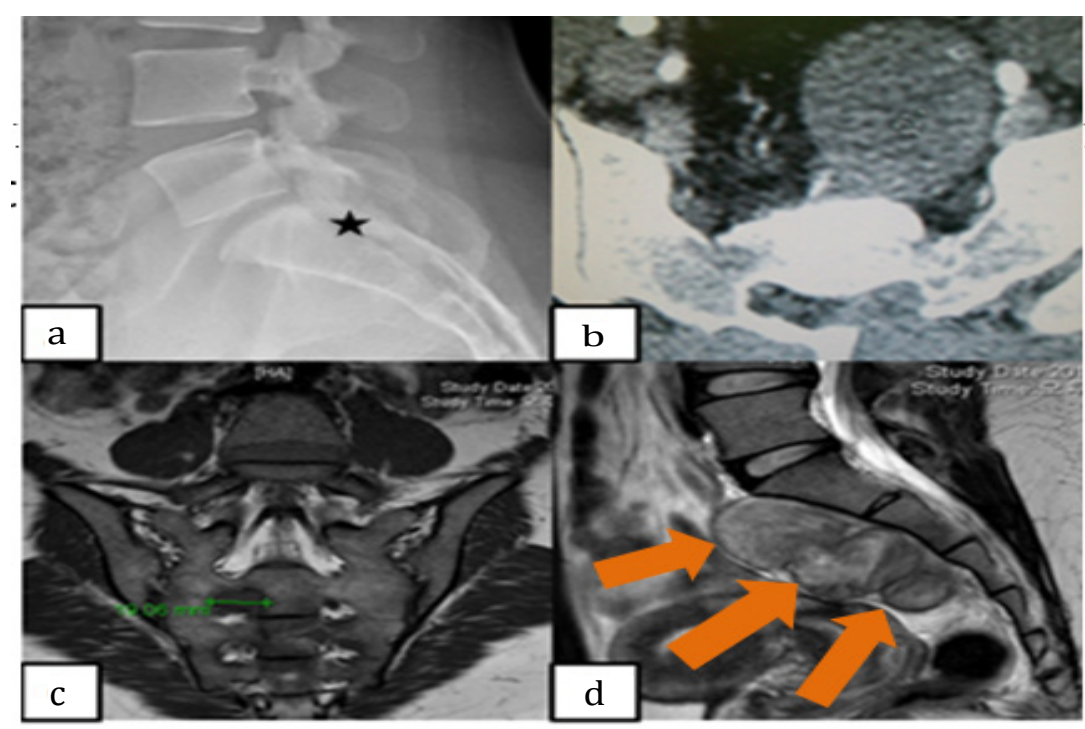

Fig. 1 a) Destruction of Os Sacrum (asterisk) b) Axial Image of NC CT-Scan Large Encapsulated and Bone Defect in Sacral Area c) Coronal Images of T2-weighted MRI Showed Foraminal Enlargement of the Sacrum d) Nonhomogenous Hyperintense Presacral Mass on T2-weighted MR Image. ${ }^{2,3}$

on its proximity to the target tissue. As of the present, the application of this technology for the excision of a presacral tumor has appeared in the literature in only one report. ${ }^{9}$ Although robotic surgery is presently an interesting and emerging technique in various surgical fields, the effectiveness of this equipment is still under debate. ${ }^{10,11}$ The goal of this article is to review the da Vinci surgical system, which is the currently most well known robotic surgical system.

\section{Case}

A 29-year-old female patient complained of a right pelvic pain for 1 month. Pelvic magnetic resonance imaging revealed the presence of a $17 \times 8 \times 10 \mathrm{~cm}$ non-homogeneous encapsulated mass with cystic change located in the pelvic cavity. This tumor had caused an anterior displacement of the urinary bladder and colon (Fig. 1).

The tumor was removed for biopsy through an open posterior laminectomy of the sacrum in prone position and, a week later, da Vinci robot procedure via transperitoneal approach in a supine position was performed. ${ }^{12}$ Once the patient was placed in a supine position, the da Vinci robot was docked and a neurosurgeon then sat by the robotic console while assistance was provided by a digestive surgeon at the side of the operating table. A $12 \mathrm{~mm}$ trocar for the camera was inserted just above the umbilicus. Two 8-mm trocars were introduced 8-10 cm away from the camera port (Fig. 2). A trocar for the right side of robotic arm was introduced and was directed to the symphysis pubis while the left side trocar was at an intersection point between the left mid-clavicular line and the imaginary line from umbilicus to left anterior superior iliac spine. Another 12-mm trocar was made for suctioning and assisting between the camera port and 8-mm trocar. The distances between the trocars were wide enough to avoid collisions of instruments (Fig. 3B). After the trocar insertion, the da Vinci Surgical System $\AA$ was introduced from the backside of the patient cart. Maryland bipolar forceps and monopolar curved scissors were employed as the left and right instruments, respectively (Fig. 3C). Detachment of the large bowel from the posterior peritoneal wall was performed. Incision of the posterior peritoneum was made and small vessels traversing the tumor capsule were coagulated with bipolar forceps 


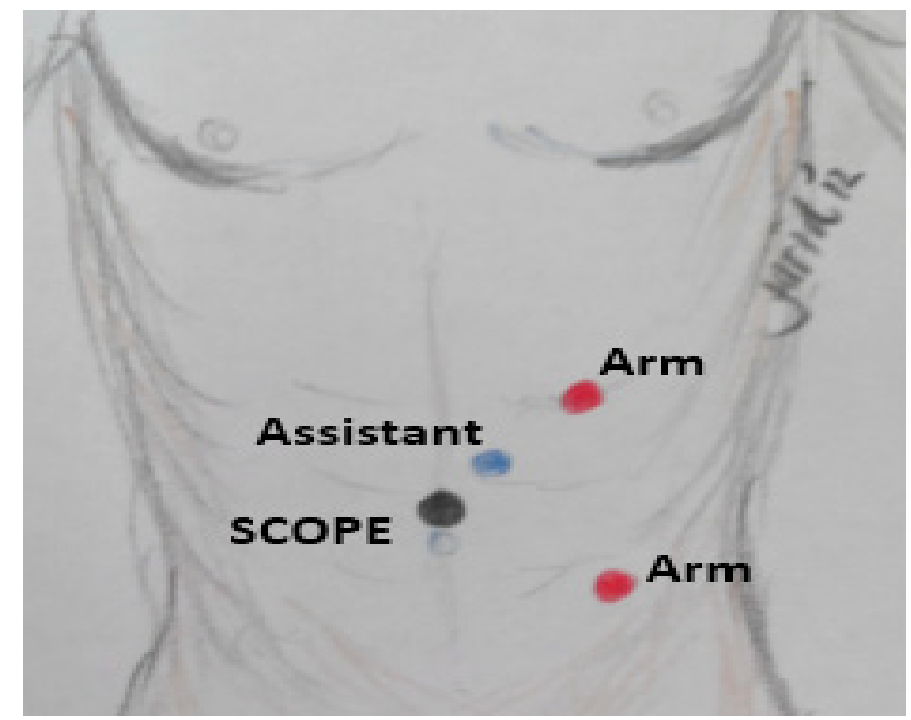

\section{Fig. 2 Artist Diagram Shows Port Placement for Robot-assisted Presacral Tumor Resection}

and incised with an activated scissors. The tumor capsule was extremely thin and friable; however, the tumor was not soft enough to be removed with suction. Considering that condition, an intracapsular tumor debulking was undertaken; tumor was resected following a circumferential dissection and excised with minimal traction on the originating nerve (Fig. 3D). A subtotal excision left a part of the capsule to preserve the nerve. The specimen was placed in the EndoCatch retrieval bag (Autosuture, Norwalk, CT, USA) and removed through the assistant port. Amount of bleeding was less than 100 cc. Setup time for the da Vinci Surgical System ${ }^{\circledR}$ was $40 \mathrm{~min}$, and the total time of anesthesia was $180 \mathrm{~min}$. The sum of all incision sizes was $8 \mathrm{~cm}$. Although bleeding was minimal, an indwelling catheter was left attached for the possibility of any delayed bleeding. The catheter was withdrawn the day after the surgery. The patient started consuming meal 6 hours following surgery and was discharged 72 hours after the surgery. $9,11,13$ Histopathologically, the tumor was composed of spindle cells growing in fascicles in a fibrillar background and consisted of verocay bodies characteristics for antoni type A schwanoma (H\&E 200x) although less cellular, which is characteristic of an antoni type B (Fig. 5). There were no systemic complication and pain remain decreased during 2 year follow up.
Table 1 Advantages and Disadvantages of Robotic Assisted Surgery ${ }^{1}$

\begin{tabular}{|c|c|}
\hline Advantage & Disadvantages \\
\hline Excellent visualization & $\begin{array}{l}\text { Abscence of tactile } \\
\text { sensation }\end{array}$ \\
\hline Improve dexterity & High cost \\
\hline Motion scaling & $\begin{array}{l}\text { Unproven clinical } \\
\text { benefit }\end{array}$ \\
\hline Tremor elimination & Demand learning curve \\
\hline $\begin{array}{l}\text { Elimination of fulcrum } \\
\text { effect }\end{array}$ & $\begin{array}{l}\text { Less flexible than } \\
\text { human }\end{array}$ \\
\hline Tele manipulation & Hand eye coordination \\
\hline \multicolumn{2}{|l|}{ Ergonomic position } \\
\hline \multicolumn{2}{|l|}{$\begin{array}{l}\text { Less infection } \\
\text { (unproven) }\end{array}$} \\
\hline $\begin{array}{l}\text { Less radiation } \\
\text { exposure }\end{array}$ & \\
\hline
\end{tabular}

\section{Discussion}

Presacral schwannomas are presumed to be arisen from a peripheral nerve in or adjacent to an anterior sacral foramen. Therefore, they are able to grow outside of the boundaries of the bone with limited degree of secondary bone 


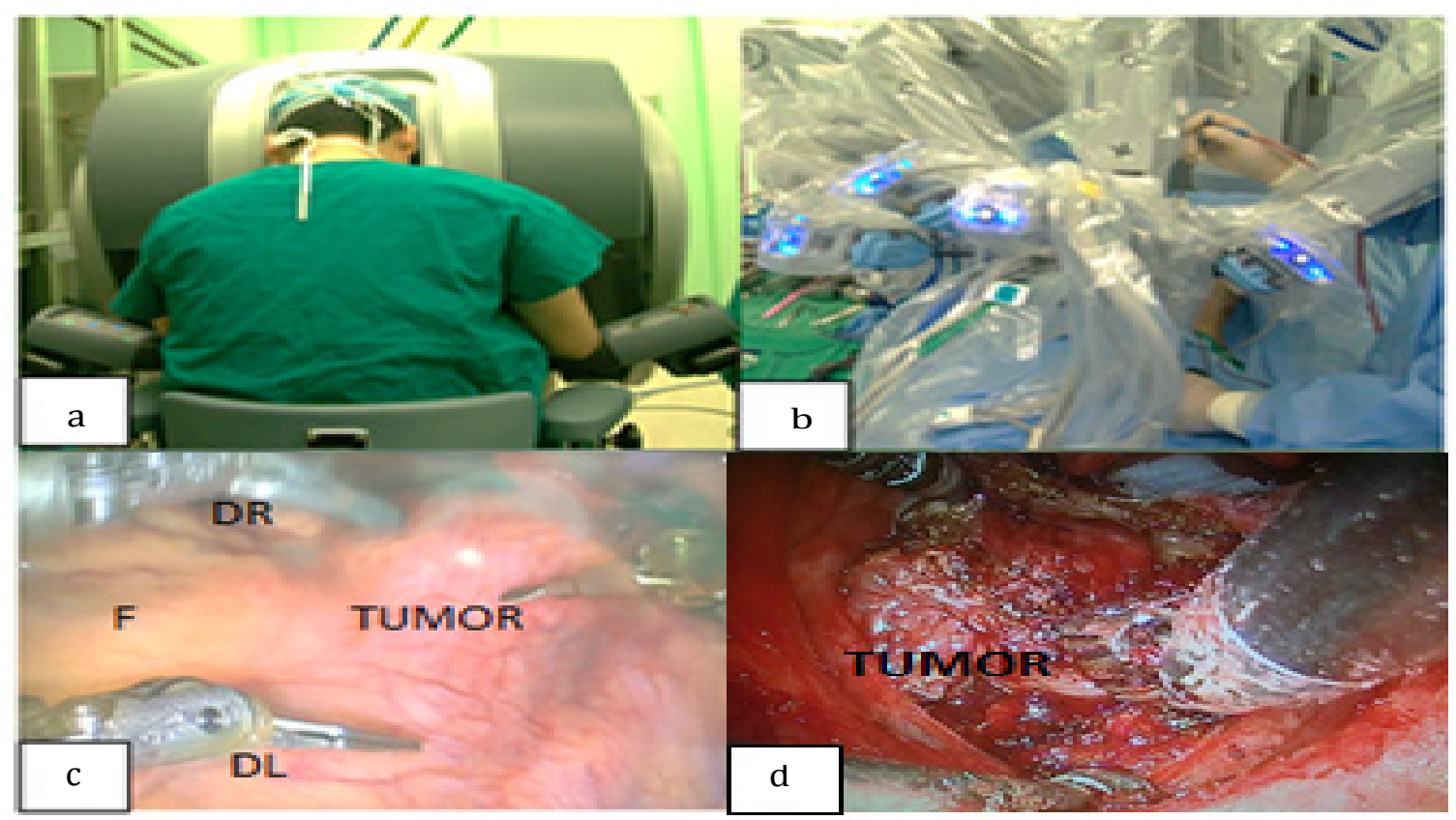

Fig. 3 Operative Findings of Robot-assisted Presacral Tumor Resection. (a) da Vinci Robot was then Docked, Neurosurgeon then Sat by the Robotic Console, (b) Colaboration with Digestive Surgeon, (c) Dissection of Tumor through Robotic Bipolar Forceps and Monopolar scissors, DL, Left Arm of da Vinci; DR, Right Arm of da Vinci; F, paracolic fat). (c) Tumor was Removed Successfully with Robot Assisted

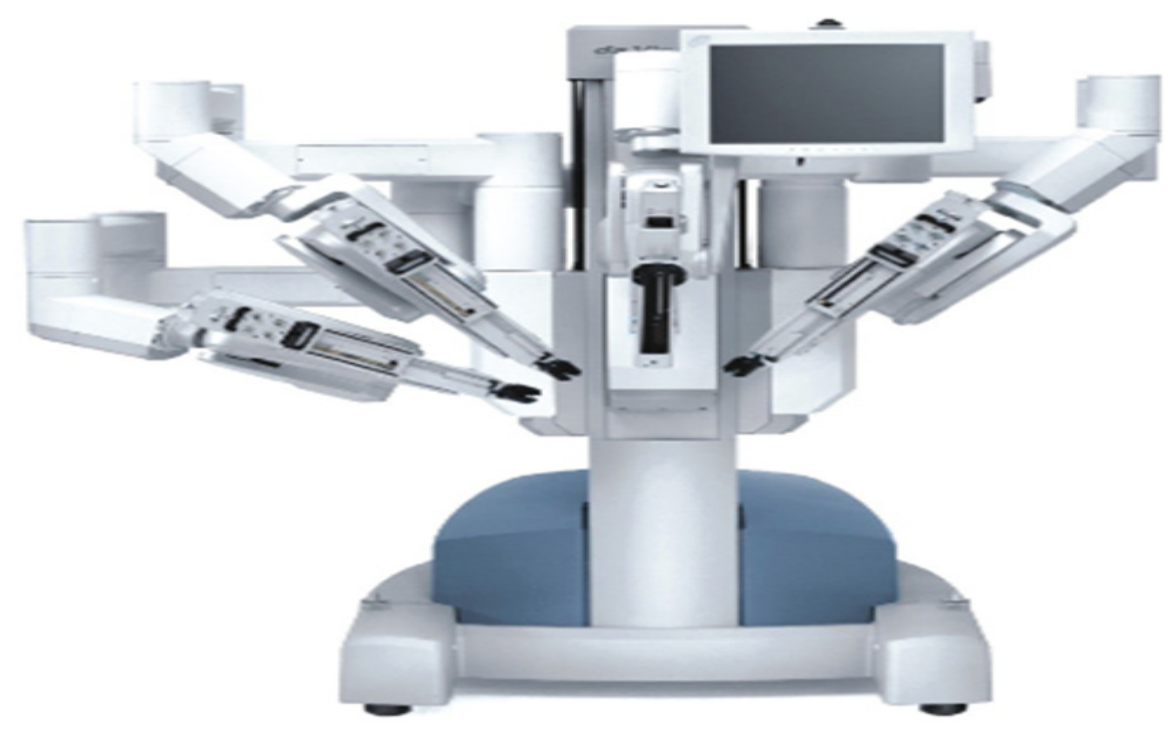

Fig. 4 da Vinci Surgical Robot System ${ }^{1}$ 


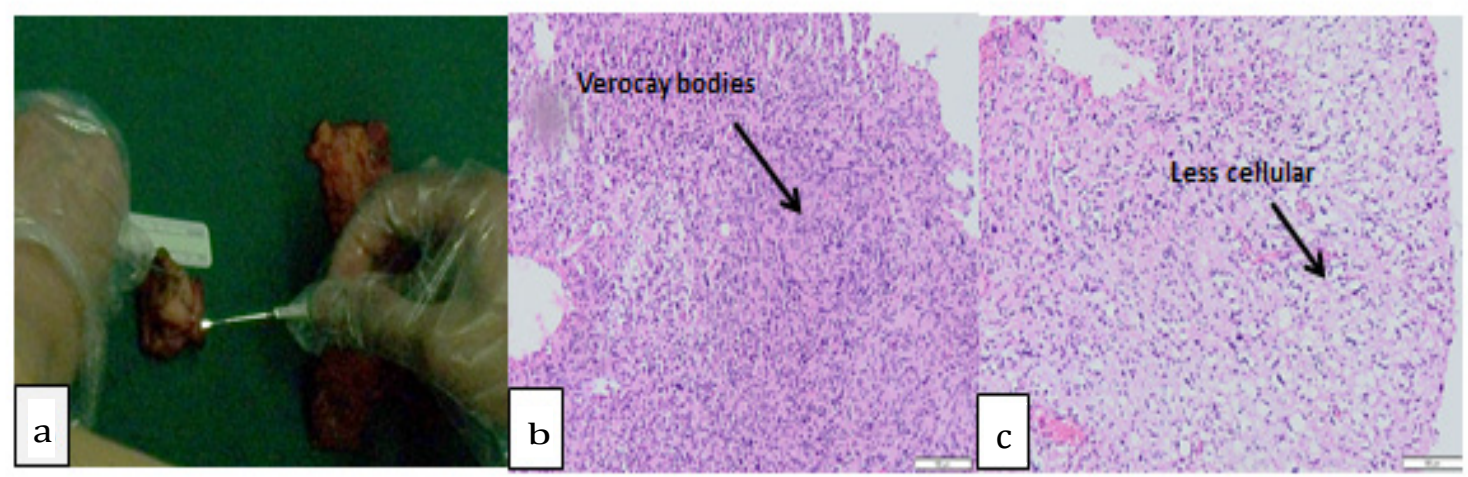

Fig. 5 a) Macroscopic Tumor Following Peacemeal Resection b) Tumor Composed of Spindle Cells Growing in Fascicles in a Fibrillar Background and Consist of Verocay Bodies and was Hypercellullar Characteristic for Antoni Type A Schwanoma (H\&E 200X). c) Hypocellularity, Characteristic for Antoni Type B. ${ }^{4,5}$

involvement with presacral retroperitoneum as the potential space. These are the possible reasons why these slowly growing tumors can develop to such a large size prior to any clinical symptoms. A presacral schwannoma is uncommon and usually asymptomatic when it is small. Retrorectal masses are typically slow growing that very mild pain in the perineal area is the most common symptom reported by patients during malignant or inflammatory processes. The tumor is often discovered after producing pain and showing neurological symptoms. ${ }^{14-16}$ Although it is rare, presacral lesions can include various types with almost $50 \%$ of presacral tumors are malignant or have areas of malignant changes within them with solid tumors having a higher probability of malignancy than the cystic lesions $(60 \%$ vs $10 \%)^{5,17}$

Magnetic resonanceimaging(MRI)technique is helpful in detecting specific characteristic and specific radiological features associated with schwannomas. The following findings are highly suggestive: a well-demarcated round or oval mass with non-homogeneous contrast enhancement due to cystic and haemorrhagic changes, presence in the region of a known nerve ganglion or pathway; calcification and cystic change in a large tumour, isoor slightly hyperintense signal intensity on T1 weighted images compared to muscle, and a high signal intensity on $\mathrm{T} 2$ weighted images. ${ }^{4,18}$ Heterogeneity can be seen on MRI imaging, which reflects degenerative change of long-standing schwannomas. There is the so-called "ancient schwannoma" referring to a degenerative schwannoma typified by cyst formation, hemorrhage, calcification, and also fibrosis. Magnetic resonance imaging can also differentiate malignant schwannomas from benign ones by identifying indistinct margins or a lack of a pseudocapsule. ${ }^{18}$

Robotic surgery has become popular for gynaecologic or urologic tumors, in spinal surgery, however, tumor outside the vertebral column such as paravertebral or presacral tumor are some of the relevant indications. Even though this operation was performed in cooperation with a laparoscopic digestive surgeon, an experienced neurosurgeon who is accustomed with the basic principles of port placement robot assisted approach shall not require a steep learning curve. ${ }^{10}$ The method still has many limitations, thus requires human assistance (Table 1). The combination of pure laparoscopic approach and the latest robotic technology enabled us to complete the first successful robotic removal of a spinal tumor with minimal morbidity, a brief hospital stay, and rapid postoperative recovery.

It can be concluded that giant presacral schwannomas are rare tumours and their surgical treatment is challenging due to the complex anatomy of the presacral. Clinical application of robotic surgery in the spinal surgical field is currently confined to some specific disease treatments or procedures. It is thefefore expected that robotic surgery will play a practical future role as a minimally invasive procedure. We believe that the advent of robotic technology will prove to be a boon to the neurosurgeon. 


\section{References}

1. Abernathey CD, Onofrio BM, Scheithauer B, Pairolero PC, Shives TC. Surgical management of giant sacral schwannoma. J Neurosurg. 1986;65(3):286-95.

2. Acciarri NS, Staffa G, Poppi M. Giant sacral schwannoma: removal by an anterior, transabdominal approach. Br J Neurosurg. 1996;10(5):489-92.

3. Haliloğlu AK, Uslu HY, Üzüm N, Haliloğlu N, Gögüş O. Giant pelvic schwannoma: a case report. Turkiye Klinikleri. J Med Sci. 2010;30(2):750-4.

4. Dede M, Yagci G, Yenen MC, Gorgulu S, Deveci MS, Cetiner S, et al. Retroperitoneal benign schwannoma: report of three cases and analysis of clinico-radiologic findings. Tohoku J Exp Med. 2003;200(2):93-7.

5. Pongsthorn C, Ozawa H, Aizawa T, Kusakabe T, Itoi E. Giant sacral schwannoma: a report of six cases. Ups J Med Sci. 2010;115(2):146-52.

6. ReganJF, Juler GL, Schmutzer KJ. Retroperitoneal neurilemoma. Am J Surg. 1977;134(1):140-5.

7. Donal JF, Baker ME, Mahony MS, Leight GS. Benign retroperitoneal schwanoma. Urology. 1988;31(4):332-4.

8. Song JY, Kim SY, ParkEG, Kim CJ, Kim do G, LeeHK, Park IY. Schwannoma in the retroperitoneum. J Obstet Gynaecol Res. 2007;33(3):371-5.

9. Moskowitz RM, Young JL, Box GN, Paré LS, Clayman RV. Retroperitoneal transdiaphragmatic robotic-assisted laparoscopic resection of a left thoracolumbar neurofibroma. JSLS. 2009;13(1):64-8.

10. Yang MS, Jung JH, Kim JM, Kim JM, Kim CH, Yi S, et al. Current and future of spinal robot surgery. Kor J Spine. 2010;7(2):61-5.
11. Yang MS, Kim KN, Yoon DH, Pennant W, Ha Y. Robot-assisted resection of paraspinal schwannoma. J Korean Med Sci. 2011;26(1):150-3.

12. Labędź W, Kubaszewski L, Adamek J. Operative treatment of schwannoma, the primary sacral tumor-case presentation. Pol Orthop Traumatol. 2012;30;77:10-6.

13. Yang MS, Yoon DH, Kim KN, Kim H, Yang JW, Yi S, et al. Robot-assisted anterior lumbar interbody fusionin a swine model in vivo test of the da vinci surgical-assisted spinal surgery system. Spine (Phila Pa 1976). 2011;36(2):E139-43.

14. Wetzel LH, Levine E. Pictorial essay. MR imaging of sacral and presacral lesions. AJR Am J Roentgenol. 1990;154(4):771-5.

15. Satake K, Matsuyama Y, Yoshihara H, Yanase M, Miura Y. Three-dimensional images for surgical plan of giant sacral schwannoma. Spinal Cord. 2004;42(6):368-70.

16. Alfieri A, Campello $M$, Broger $M$, Vitale $M$, Schwarz A. Low-back pain as the presenting sign in a patient with a giant, sacral cellular schwannoma: 10-year follow-up. J Neurosurg Spine. 2011;14(2):167-71.

17. Aranda-Narváez JM, González-Sánchez AJ, Montiel-Casado C, Sánchez-Pérez B, JiménezMazure C, Valle-Carbajo M, et al. Posterior approach (Kraske procedure) for surgical treatment of presacral tumors. World J Gastro Surg. 2012;4(5):126-30.

18. Ozturk C, Mirzanli C, Karatoprak O, Tezer M, Aydogan M, Hamzaoglu A. Giant sacral schwannoma: a case report and review of the literature. Acta Orthop Belg. 2009;75(5):70510. 